Supplementary Information for:

\title{
Polymerization in Surfactant Liquid Crystalline Phases
}

Mohan N. Wadekar, Renu Pasricha ${ }^{\dagger}$, Anil B. Gaikwad ${ }^{\ddagger}$ and Guruswamy Kumaraswamy*

National Chemical Laboratory, Pune 411008, India.

\section{HPLC of surfactants used:}

HPLC of the surfactants was performed using a C8 column and methanol as the carrier. We used a UV-detector, and the analysis was carried out at $230 \mathrm{~nm}$. HPLC (Figures S13 ) reveal that the surfactants are not pure, but comprise of mixtures. The purity is low in the case of $\mathrm{C}_{18} \mathrm{E}_{10}$ where the HPLC chromatogram comprises a number of peaks with no dominant peak, but is higher in the case of $\mathrm{C}_{12} \mathrm{E}_{3}$ that has a purity of around $83 \%$.

\section{Characterization of the surfactant mesophase:}

The phase behaviour of the surfactant-water mixtures used in this work have been investigated in the literature. ${ }^{1}$ The surfactant-water concentrations that we use to prepare hexagonal $\left(\mathrm{C}_{12} \mathrm{E}_{9}\right.$-water $)$ and lamellar $\left(\mathrm{C}_{18} \mathrm{E}_{6}\right.$-water $)$ mesophases accord well with literature reports ${ }^{1}$ and are not close to phase boundaries with other phases. Liquid crystalline surfactant mesophases can be identified by the characteristic textures that they show in polarized optical microscopy and, by the peaks in their X-ray diffraction patterns. Polarized optical microscopy of our systems show birefringence, but due to kinetic constraints on the mobility of our high viscosity phases, we do not clearly observe, for example, the fan-shaped texture that is characteristic of the hexagonal phase. The optical micrographs obtained in our work are comparable with those published in the literature. $^{2}$ The ratio of primary and higher order peaks in the X-ray diffraction data is definitive evidence of the mesophase. For example, for hexagonal phases, the second order peak appears at a $d$-spacing corresponding to $1 / \sqrt{3}$ times the $d$-spacing of the

\footnotetext{
* Author to whom correspondence should be addressed: Polymer Science and Engineering Division, National Chemical Laboratory, Pune 411008, INDIA, Phone: 91-20-2589-3382, Fax: 91-20-2589-3234, guru@poly.ncl.res.in

TEM facility, Center for Materials Characterization, NCL

$\$$ SEM facility, Center for Materials Characterization, NCL
} 


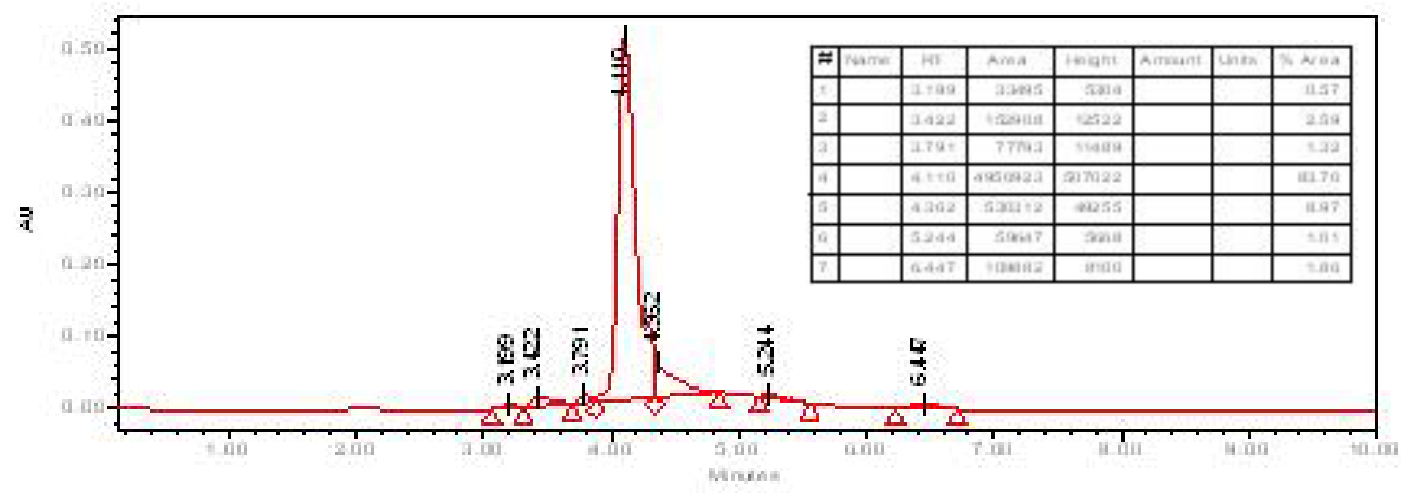

Figure S1: HPLC chromatogram of $\mathrm{C}_{12} \mathrm{E}_{9}$

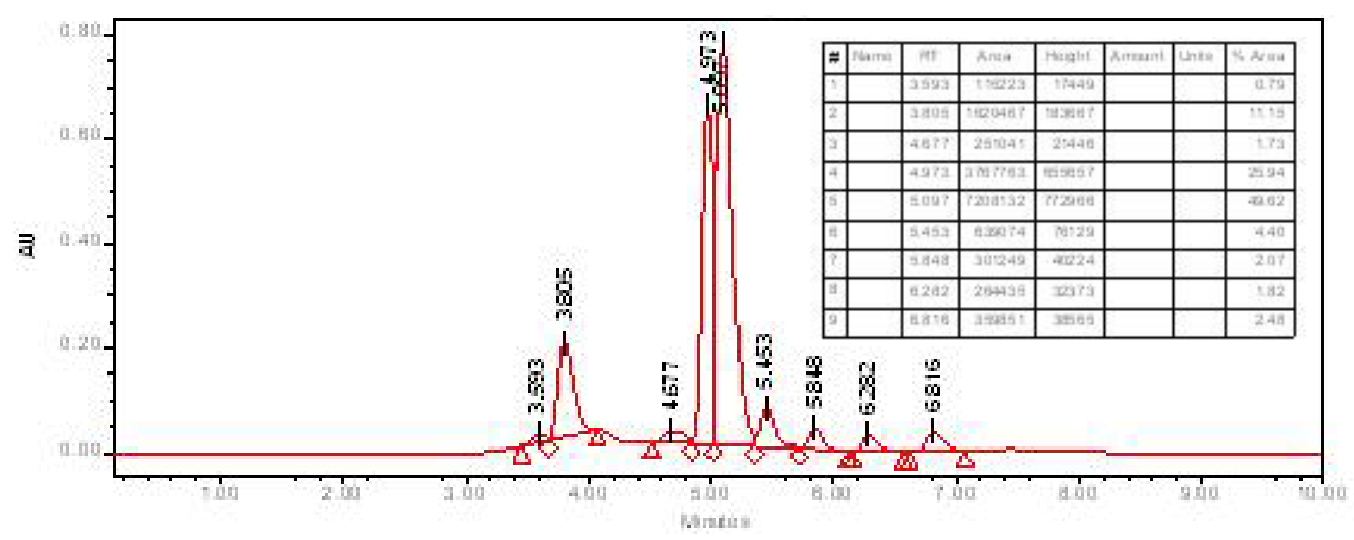

Figure S2: HPLC chromatogram of $\mathrm{C}_{18} \mathrm{E}_{2}$

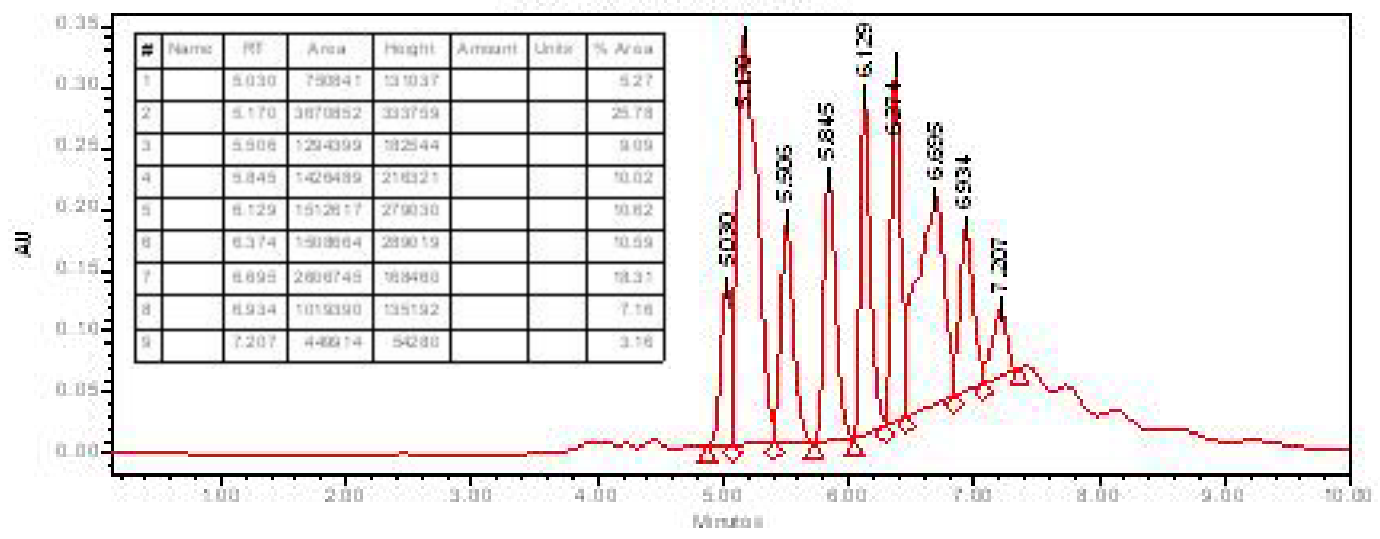

Figure S3: HPLC chromatogram of $\mathrm{C}_{18} \mathrm{E}_{10}$

primary peak. We observe these characteristic second order peaks in our $\mathrm{C}_{12} \mathrm{E}_{9}$-water hexagonal phases (Figure S4), not only for the neat hexagonal phase, but also for the hexagonal phase in which the DMS and TMS monomers have been condensed. 


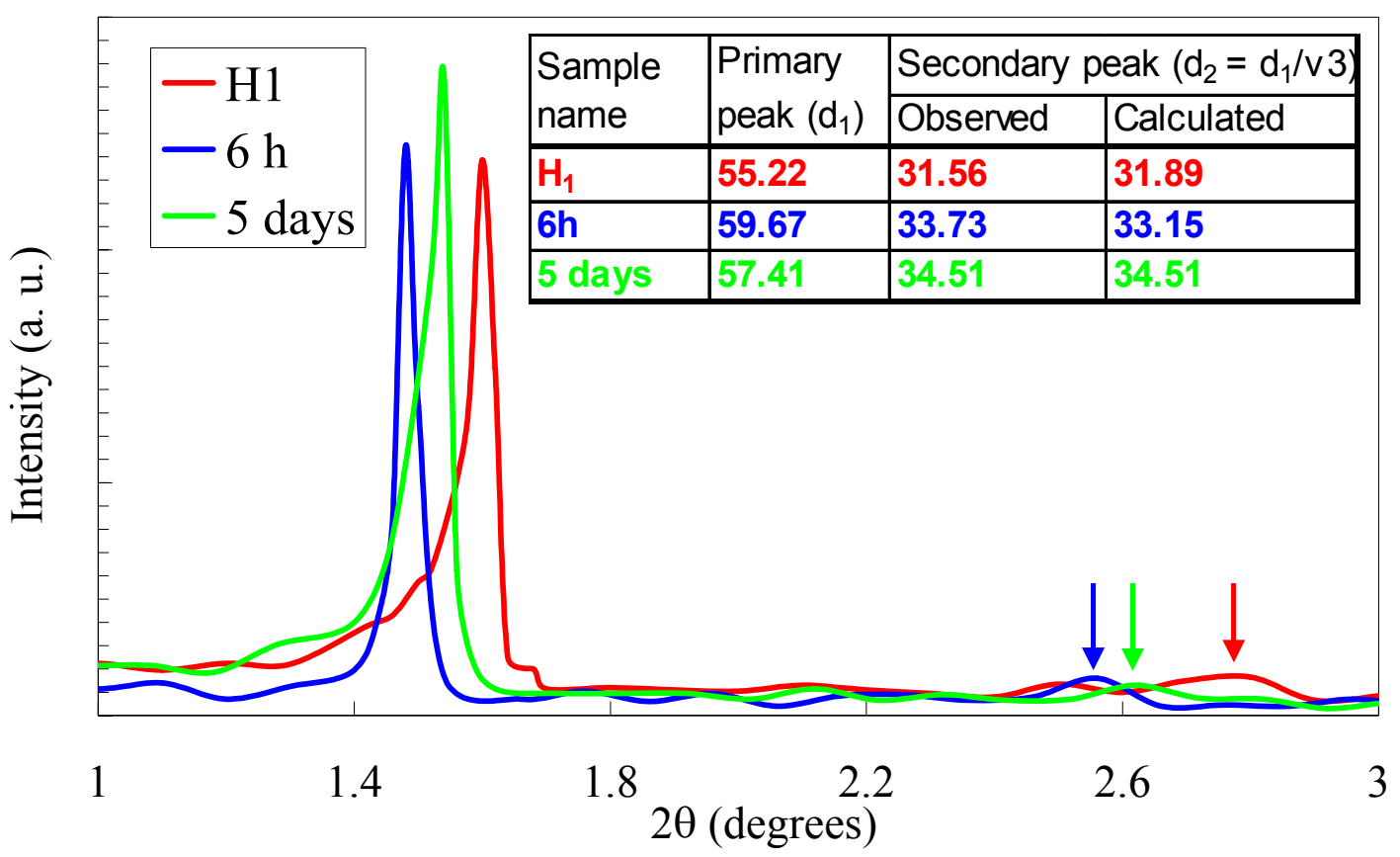

Figure S4: X-ray diffraction patterns showing the primary and secondary peaks for the $\mathrm{C}_{12} \mathrm{E}_{9}$-water system in the hexagonal phase. Intensity is plotted on a linear scale. We observe second-order peaks for the neat hexagonal phase as well as after polymerization of the DMS and TMS monomers (as explained in the main paper) for a period of 6 hours and 5 days.

\section{Loading the surfactant mesophase:}

The surfactant mesophases described in this work swell on being loaded with organic solvents or monomers such as DMS. These organics are localized in the hydrophobic parts of the surfactant assembly and the d-spacing observed in the X-ray diffraction pattern increases. Beyond a certain concentration of the loaded monomer, the mesophase is destabilized and transitions to another phase. This limiting value of the monomer loading is between 8 to $12 \%$ for the hexagonal phase and about 6 to $8 \%$ for the lamellar phase examined here. Figure S5 shows the increase in d-spacing when p-xylene is added to the $\mathrm{C}_{12} \mathrm{E}_{9}$-water hexagonal phase. We see that the increase in $\mathrm{d}$-spacing with added $\mathrm{p}$ xylene is approximately linear. Beyond a p-xylene volume fraction of about $10 \%$, the $\mathrm{X}$ ray diffraction peaks corresponding to the hexagonal phase disappear and an oily micellar phase is obtained. The surfactant phase is no longer a high viscosity gel, but transitions to a low viscosity fluid. 


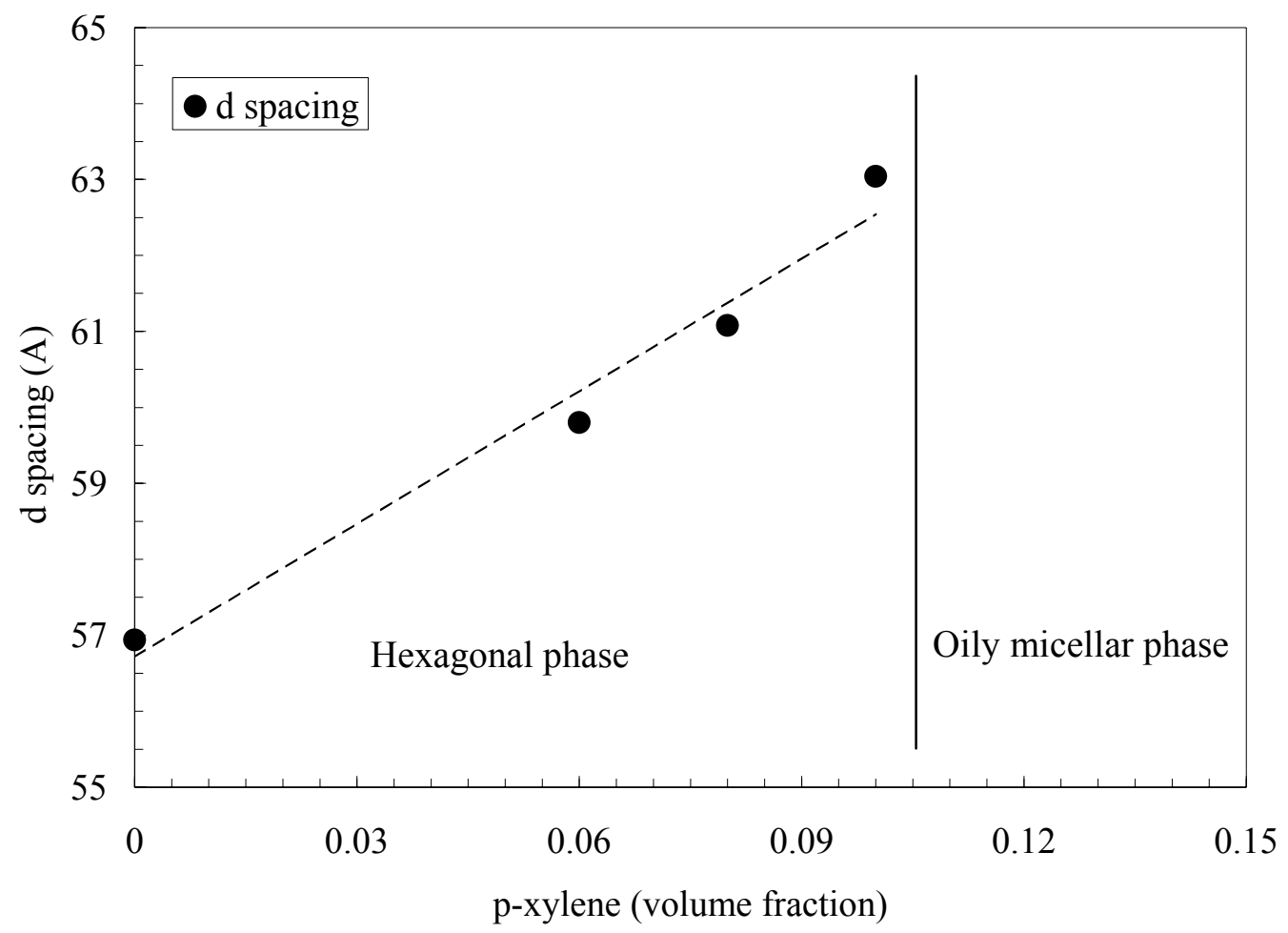

Figure S5: Increase in d-spacing observed in X-ray diffraction upon addition of p-xylene to the hexagonal phase of the $\mathrm{C}_{12} \mathrm{E}_{9}$-water system. A linear fit is shown as a guide to the eye. The hexagonal phase persists until a p-xylene volume fraction of about $10 \%$, and then transitions to a low viscosity oily micellar phase.

\section{Condensation of TMS in dodecane-0.2M sulphuric acid system:}

When TMS is added to a dodecane-acidified water system, the TMS initially goes into the organic phase. After about 15 minutes, the aqueous phase turns hazy as TMS hydrolyses and starts condensing (Figure S6 top left). With time, the TMS condenses in the aqueous phase until it forms a cross-linked film that then phase separates and is localized at the water-dodecane interface (Figure S6, bottom right). We isolated this film, dried it and prepared a sample for FTIR by mixing with $\mathrm{KBr}$ powder. FTIR was performed on this powder in diffuse reflectance mode on a Perkin-Elmer Spectrum-1. IR Absorbance at $772 \mathrm{~cm}^{-1}$ confirms that the film contains $\mathrm{Si}-\mathrm{CH}_{3}$ bonds while absorbances at $1035 \mathrm{~cm}^{-1}$ and at $1118 \mathrm{~cm}^{-1}$ are indicative of an open chain siloxane network (Figure S7). 

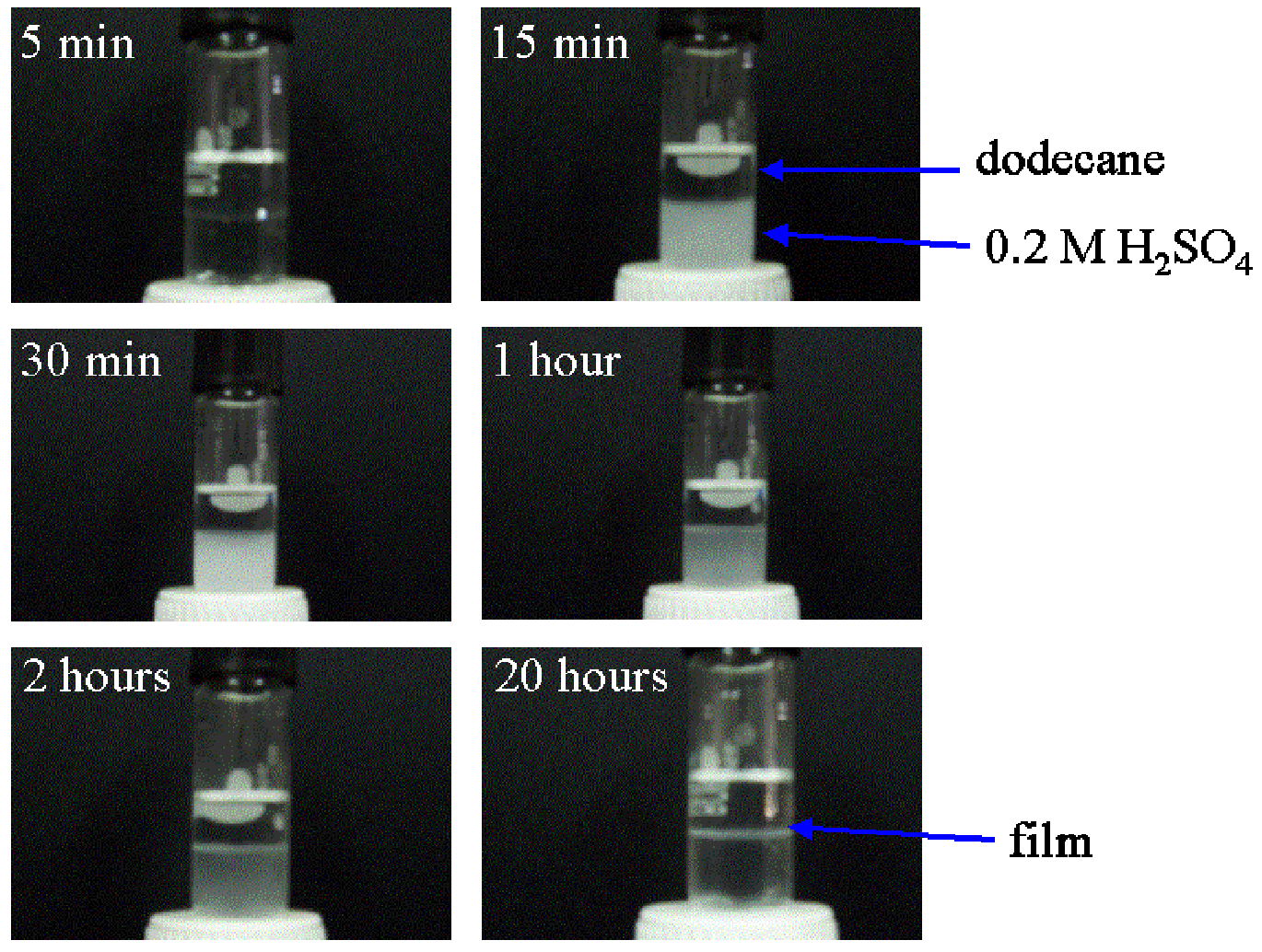

Figure S6: Digital photographs of TMS condensation in a dodecane-acidified water system. Dodecane is lighter than water and forms the top phase. The formation of the cross-linked TMS film is observed in the final photograph taken 20 hours after the addition of TMS.

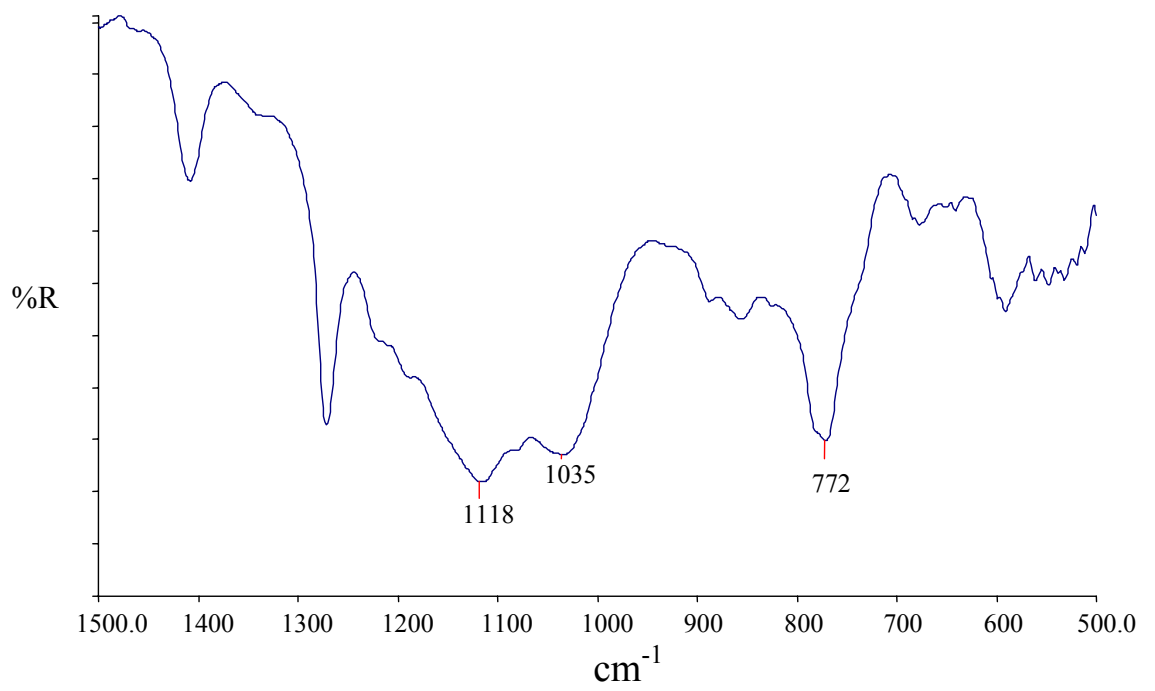

Figure S7: FTIR of cross-linked TMS film observed in Figure S6. 


\section{Characterization of the polysiloxanes synthesized in the mesophase:}

The rod-like polymer particles prepared by condensation of DMS and TMS mixtures in the hexagonal mesophase were separated from the surfactants by washing. We dispersed the surfactant gels containing particles in acetone, and pipetted out the supernatant after allowing the polysiloxane particles to settle. We repeatedly washed the particles with chloroform, collected them and performed FTIR on them in a $\mathrm{KBr}$ cell. The FTIR clearly shows the presence of characteristic polysiloxane peaks at $1090,1020 \mathrm{~cm}^{-1}$ corresponding to Si-O-Si stretching and at $1262 \mathrm{~cm}^{-1}$ corresponding to symmetrical methyl deformation (Figure S8).

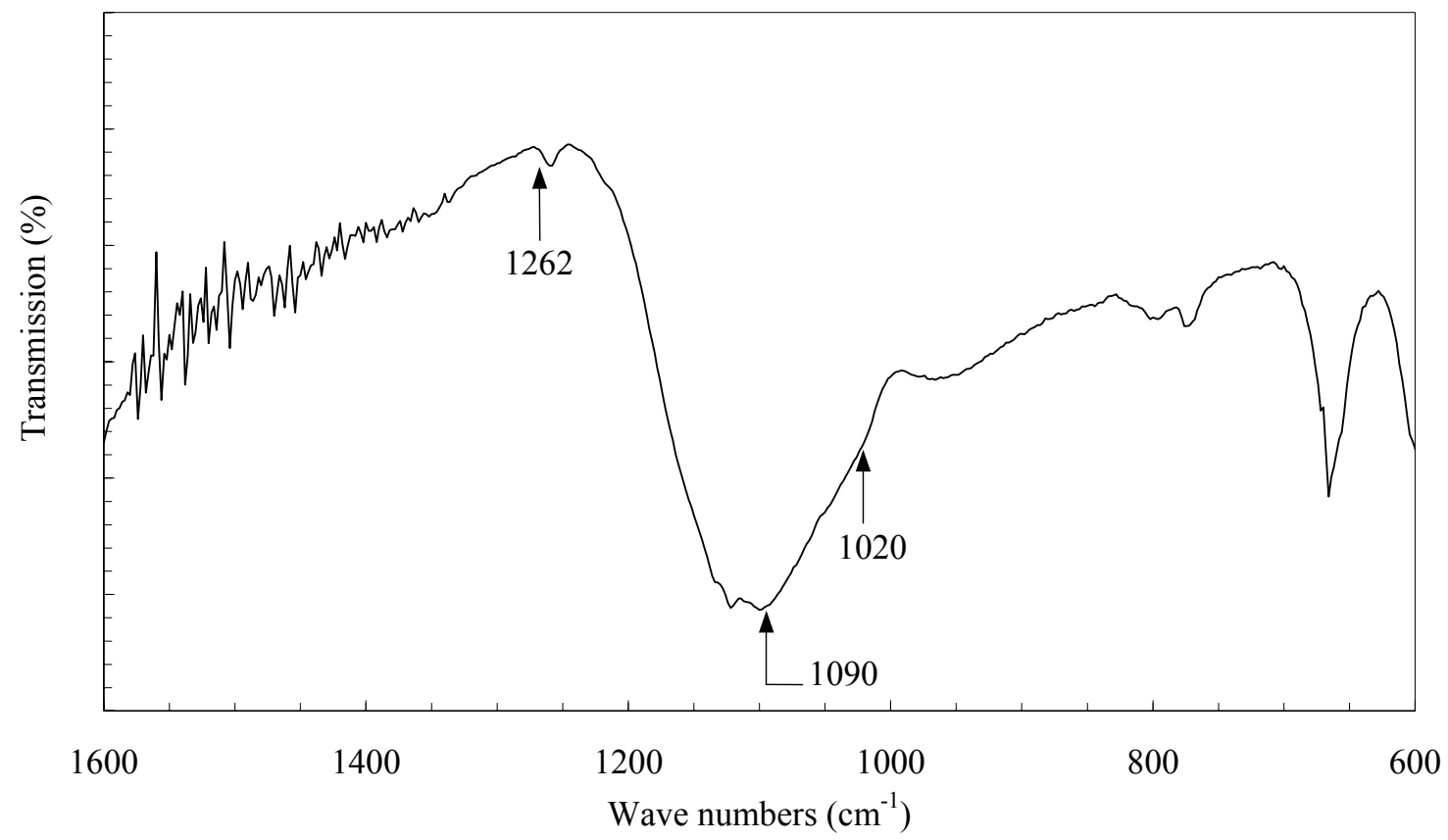

Figure S8: FTIR spectrum for rod-like polysiloxane separated from the surfactant mesophase. As the volume of polysiloxane isolated was small, the spectrum has poor signal to noise ratio - however, the characteristic peaks for the polysiloxanes are clearly visible above the noise. 

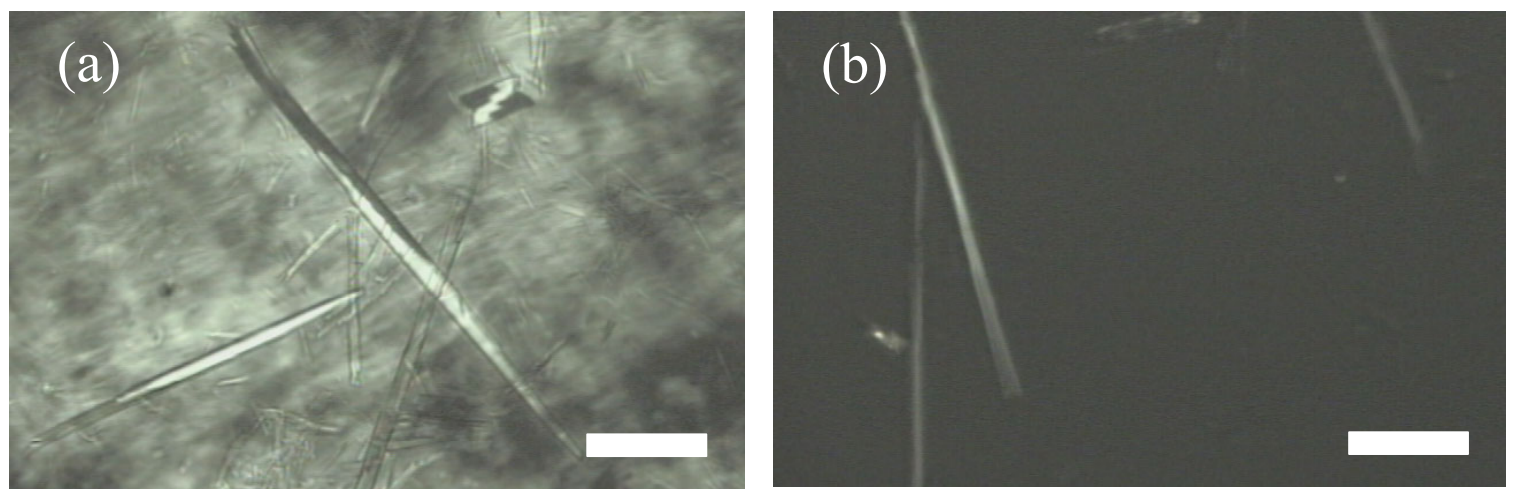

Figure S9: Optical micrographs of polysiloxane rods in the $\mathrm{C}_{12} \mathrm{E}_{9}$-water hexagonal mesophase observed under crossed-polarizers. The first micrograph (a) is taken at $30^{\circ} \mathrm{C}$, below the clearing temperature of the mesophase while (b) is taken at $45^{\circ} \mathrm{C}$, above the clearing temperature. As the surfactant flows at $45^{\circ} \mathrm{C}$, the rods have translated in (b) relative to their position in (a). The scale bar corresponds to $50 \mu \mathrm{m}$.

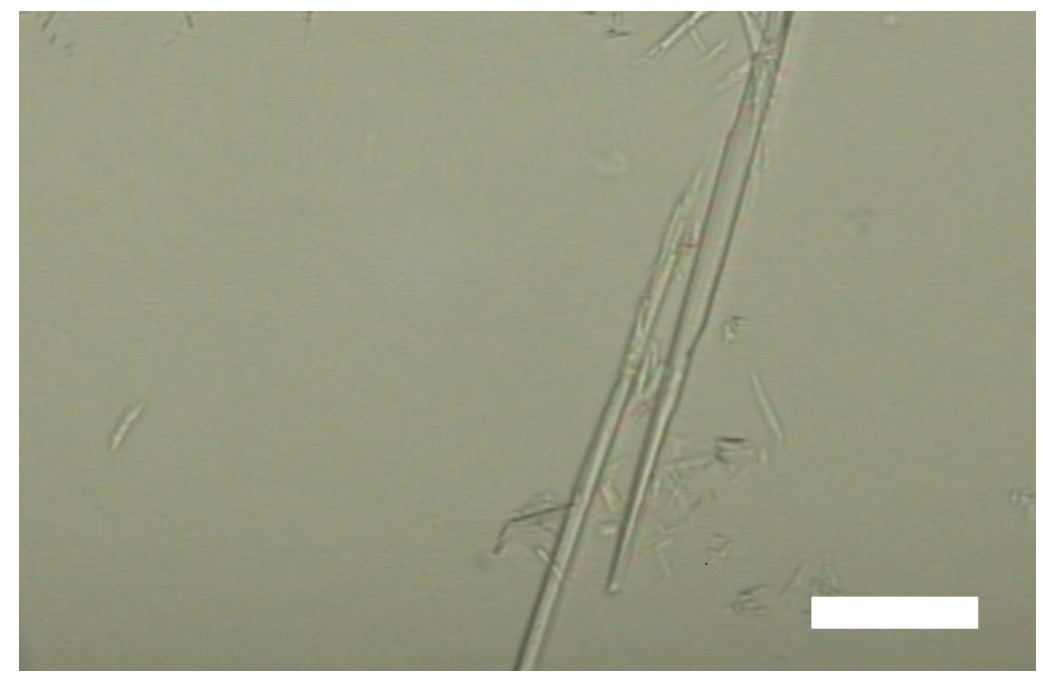

Figure S10: Optical micrograph of polysiloxane particles surrounded by the surfactantwater phase at a temperature of $80^{\circ} \mathrm{C}$. The scale bar corresponds to $50 \mu \mathrm{m}$.

Further, when the surfactant gel containing polysiloxane rods is heated in the polarized optical microscope using a Mettler hotstage, we observe that the rods are stable even above the clearing point of the surfacant mesophase (about $40^{\circ} \mathrm{C}$, Figure S10). Below the clearing point of the surfactant mesophase, the characteristic birefringence associated with the liquid crystalline phase is observed in the background, around the polysiloxane 
rods (Figure S9a). However, on heating above the clearing temperature, this birefringence disappears but the rods are stable (Figure S9b). The polymer rods are stable and do not distort even on heating to a temperature of $80^{\circ} \mathrm{C}$, suggesting that the polysiloxane that constitutes the rods is highly cross-linked (Figure S10). The data presented in this section (the FTIR and the optical micrographs) conclusively prove that the particles observed in our experiments comprise of cross-linked polysiloxanes that are stable much above room temperature.

\section{References:}

(1) Kunieda, H.; Shigeta, K.; Ozawa, K.; Suzuki, M. J. Phys. Chem. B 1997, 101, 7952.

(2) Hentze, H-P.; Kaler, E. W. Chem. Mater. 2003, 15, 708. 